\title{
100-Gb/s Transmission Using Orthogonal Frequency-Division Multiplexing
}

\author{
Ivan B. Djordjevic, Member, IEEE, and Bane Vasic, Senior Member, IEEE
}

\begin{abstract}
The possibility of 100-Gb/s transmission over 25-GHz bandwidth using orthogonal frequency-division multiplexing (OFDM) is demonstrated. It is shown that $100-\mathrm{Gb} / \mathrm{s}$ transmission over $3840 \mathrm{~km}$ can be achieved using single-sideband quadrature-phase-shift keying OFDM transmission and low-density parity-check codes.
\end{abstract}

Index Terms-100-G Ethernet, high-speed optical transmission, optical communications, orthogonal frequency-division multiplexing (OFDM).

\section{INTRODUCTION}

$\mathbf{E}$ THERNET was initially introduced as a communication standard for short-distance connection of hosts in a local area network (LAN) [1]. Due to low cost, high speed, and simplicity compared to other protocols, it has rapidly evolved, and has already been used in campus-size distance connections, and beyond, in metropolitan area networks [1], [2]. The network interface cards for 1 - and $10-\mathrm{Gb} / \mathrm{s}$ Ethernet are already commercially available [2]. Traditionally, the Ethernet data rates have grown in $10-\mathrm{Gb} / \mathrm{s}$ increments, so the data rate of $100 \mathrm{~Gb} / \mathrm{s}$ is being envisioned as the speed of the next generation of Ethernet [1]-[3]. The 100-Gb/s all-electrically time-division-multiplexed (ETDM) transponders are becoming increasingly important [3]-[6] because they are viewed as a promising technology that may be able to meet speed requirements of the new generation of Ethernet. Despite the recent progress in high-speed electronics [6], ETDM modulators and photodetectors are still not widely available, so that alternative approaches to achieving a $100-\mathrm{Gb} / \mathrm{s}$ transmission using commercially available components and differential quadrature-phase-shift keying (QPSK) is of great current interest [4].

In this letter, we propose an alternative technique to QPSK and $100-\mathrm{Gb} / \mathrm{s}$ ETDM, which allows us to achieve a $100-\mathrm{Gb} / \mathrm{s}$ optical transmission using commercially available components. It is based on the orthogonal frequency-division multiplexing (OFDM) [7]. OFDM is a special case of multicarrier transmission in which a single information-bearing stream is transmitted over many lower rate subchannels. It has been used for digital audio broadcasting [7], high-definition television (HDTV) terrestrial broadcasting [8], in high bit-rate digital subscriber line, asymmetric DSL and very high data rate DSL [7], IEEE 802.11, high-performance LAN type 2 (HIPERLAN/2) and multimedia mobile access communications wireless LANs [7], radio-over-

Manuscript received March 27, 2006; revised May 9, 2006. This work was supported in part by the National Science Foundation (NSF) under Grant ITR 0325979 and Grant CCR 0208597.

The authors are with the Department of Electrical and Computer Engineering, University of Arizona, Tucson, AZ 85721 USA (e-mail: ivan@ece.arizona.edu).

Digital Object Identifier 10.1109/LPT.2006.879590 fiber-based networks [9], and for free-space optics communications [10]. OFDM offers good spectral efficiency and high immunity to chromatic dispersion. Our OFDM system has 64 subcarriers, and a bandwidth of $25 \mathrm{GHz}$. Fifty of these carriers are used for carrying $2 \times 1 \mathrm{~Gb} / \mathrm{s}$ Ethernet traffic using either QPSK or 16-quadrature-amplitude modulation (16-QAM), and the remaining 16 subcarriers are used for transmission of pilot signals and for the forward-error correction (FEC) overhead. We show that four pilots are sufficient to mitigate the influence of SPM, which means that an aggregate capacity of $120 \mathrm{~Gb} / \mathrm{s}$ is possible.

\section{100-Gb/s TRANSMISSION USING OFDM}

The basic OFDM transmitter and receiver configurations are given in Fig. 1(a) and (b), respectively. $b_{i} 1-\mathrm{Gb} / \mathrm{s}$ data streams are mapped into a two-dimensional signal point from a $2^{\text {bi }}$-point signal constellation such as QAM, which is considered in this letter. The complex-valued signal points from all $K$ subchannels are considered as the values of the discrete Fourier transform (DFT) of a multicarrier OFDM signal. The symbol interval length in an OFDM system is $T=K T_{s}$, where $T_{s}$ is the symbol-interval length in a single-carrier system. By selecting $K$, the number of subchannels, sufficiently large, the OFDM symbol interval can be made much larger than the dispersed pulsewidth in a single-carrier system, resulting in an arbitrary small intersymbol interference. The OFDM symbol, shown in Fig. 1(c)-(d), is generated as follows: $N_{\mathrm{QAM}}$ input QAM symbols are zero-padded to obtain $N_{\mathrm{FFT}}$ input samples for inverse fast Fourier transform (IFFT), the $N_{\text {guard }}$ samples are inserted to create the guard interval $T_{\text {guard }}$, and the OFDM symbol is multiplied by the window function (raised cosine function is used in [7], however, the Kaiser, Blackman-Harris, and other window functions are also applicable). The purpose of cyclic extension is to preserve the orthogonality among subcarriers even when the neighboring OFDM symbols partially overlap due to dispersion, and the purpose of the windowing is to reduce the out-of-band spectrum. The cyclic extension, illustrated in Fig. 1(c), is done by repeating the last $N_{\text {guard }} / 2$ samples of the effective OFDM symbol part (of duration $T_{\mathrm{FFT}}$ with $N_{\mathrm{FFT}}$ samples) as the prefix, and repeating the first $N_{\text {guard }} / 2$ samples (out of $N_{\mathrm{FFT}}$ ) as the suffix. (Notice that windowing is more effective for smaller numbers of subccarriers.) For example, for QPSK OFDM transmission $b_{i}=21-\mathrm{Gb} / \mathrm{s}$ streams create a QPSK signal constellation point; with 50 subcarriers carrying $2 \mathrm{~Gb} / \mathrm{s}$, the aggregate rate of $100 \mathrm{~Gb} / \mathrm{s}$ is achieved. After a digital-to-analog conversion and RF up-coversion, the OFDM signal is driven to the Mach-Zehnder modulator (MZM) and then transmitted over the fiber. The dc component is inserted to be able to recover the QAM symbols incoherently. It is assumed that $50 \%$ of the total launched power (observed in electrical domain) is allocated for the transmission of carrier. To improve the 


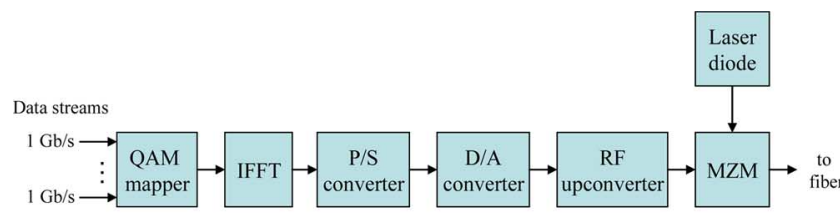

(a)

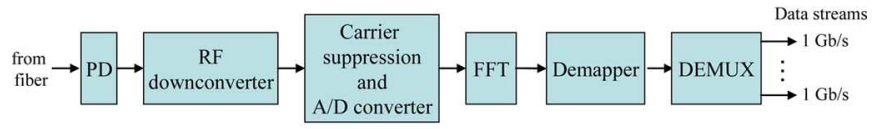

(b)

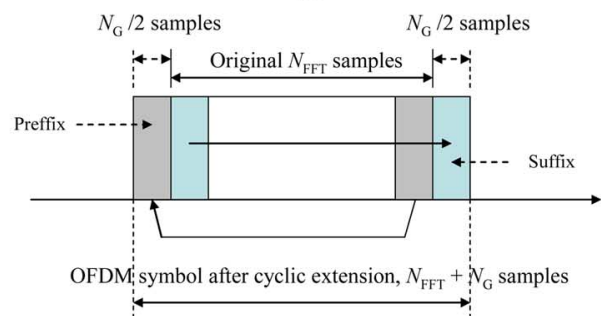

(c)

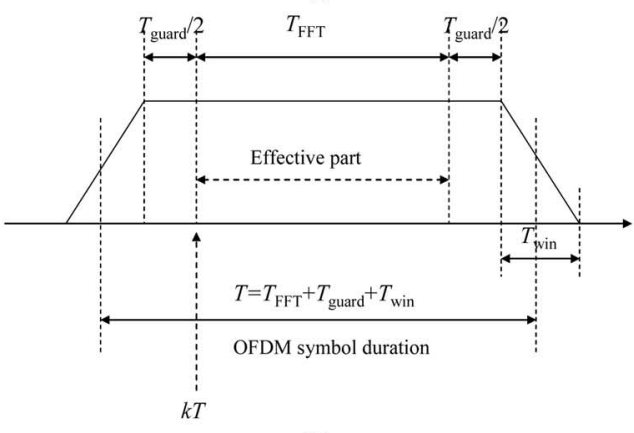

(d)

Fig. 1. (a) Transmitter and (b) receiver configurations; (c) OFDM symbol cyclic extension; (d) OFDM symbol after windowing. (Color version available online at http://ieeexplore.ieee.org.)

power efficiency a single-sideband (SSB) OFDM transmission is achieved. The information is imposed by modulating the electrical field of continuous-wave laser beam by using the OFDM signal as RF input of an MZM. At the receiver side, after the photodetection (PD), RF down-conversion and carrier suppression, the received signal is demodulated by computing the DFT.

For demonstration purposes, the power spectral densities (PSDs) after MZM are shown in Fig. 2(a) and (b) for double-sideband (DSB) transmission, and SSB transmission, respectively. The PSD at photodetector output for SSB transmission is shown in Fig. 2(c). The in-phase component of RF signal after up-conversion is given in Fig. 2(d), and shows that MZM operates in linear regime. The OFDM signal bandwidth is set to $25 \mathrm{GHz}$, the number of subchannels is set to $N_{\mathrm{QAM}}=64$, FFT/IFFT is calculated in $N_{\mathrm{FFT}}=128$ points, $\mathrm{RF}$ carrier frequency is set to $60 \mathrm{GHz}$, the bandwidth of optical filter is set to $120 \mathrm{GHz}$, and the total averaged launched power is set to $0 \mathrm{dBm}$. One hundred data strings carrying 1-Gb/s Ethernet traffic are QPSK or 16-QAM modulated, and transmitted on 50 subcarriers. The remaining 14 subcarriers are used for the transmission of pilots and FEC overhead. The guard interval is obtained by cyclic extension of $N_{\text {guard }}=2 \times 16$ samples as explained above, and the windowing ( $N_{\text {win }}=2 \times 16$ samples $)$ is based on Blackman-Harris windowing function.
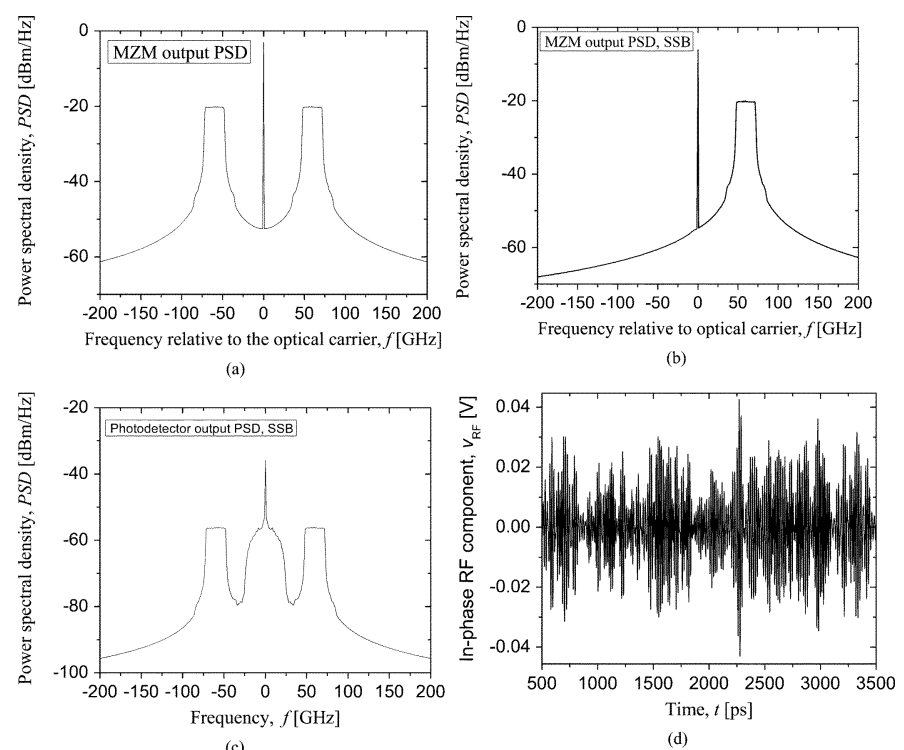

Fig. 2. PSD for (a) DSB and (b) SSB transmission. (c) PD output PSD for SSB transmission. (d) In-phase RF up-conversion waveform.

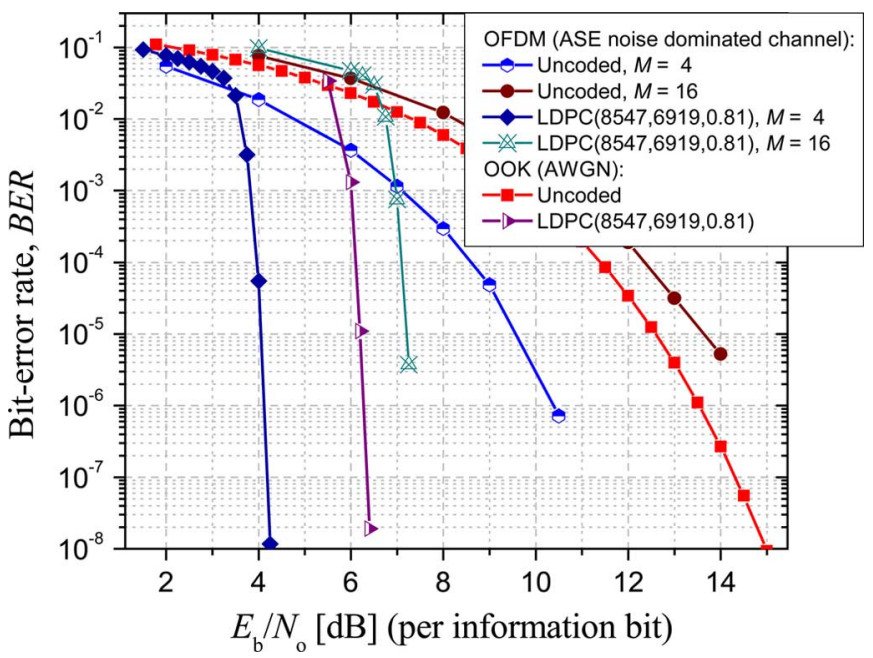

Fig. 3. BER of OFDM system versus OOK for ASE noise dominated channel. (Color version available online at http://ieeexplore.ieee.org.)

Fig. 3 shows the comparison of the bit-error-rate (BER) performances of the ON-OFF keying (OOK) system and the OFDM transmission over the amplified spontaneous emission (ASE) noise dominated channel. Because the OFDM is essentially an RF coherent scheme, it provides an initial 3-dB improvement compared to OOK. However, an initial 3-dB advantage is lost due to transmission of dc bias. Moreover, for the same launched powers (into fiber), the energy per bit for return-to-zero (RZ)-OOK is significantly larger than that of OFDM. This is the only way to keep fiber nonlinearities reasonably low, and to allow MZM and RF amplifier to operate in the linear regime. The comparison is done in the electrical domain (see [7] for definition of signal-to-noise ratio) because the concept of optical signal-to-noise ratio is not straightforward to define for OFDM waveforms [see Fig. 2(d)]. The FEC is based on the low-density parity-check (LDPC) (8547, 6919) code of code rate 0.81 proposed by the authors in [11]. As it has been shown in [11], the employed LDPC code does 


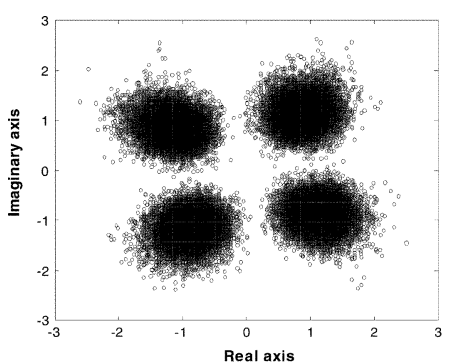

(a)

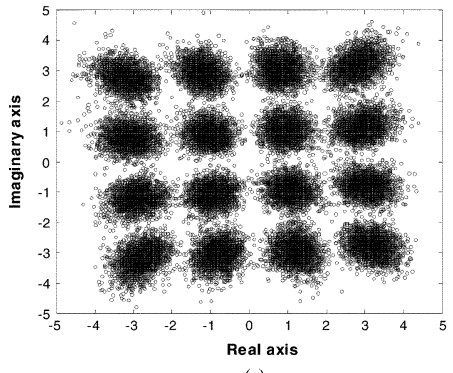

(c)

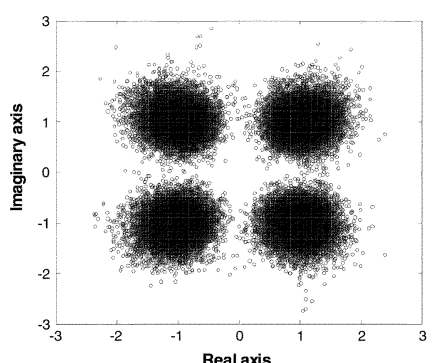

(b)

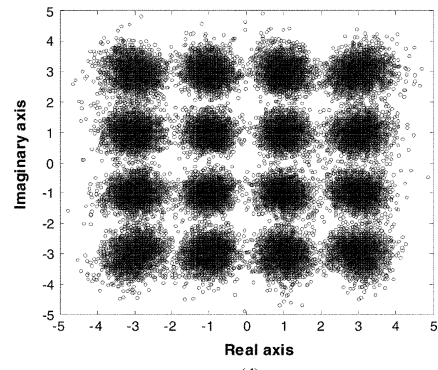

(d)
Fig. 4. Received signal constellation for QPSK SSB transmission after 20 spans: (a) before phase correction, (b) after phase correction. The received signal constellation for 16-QAM SSB transmission after 10 spans: (c) before phase correction, (d) after phase correction. Average launched power is set to $-3 \mathrm{dBm}$, and erbium-doped fiber amplifier noise figure to $6 \mathrm{~dB}$.

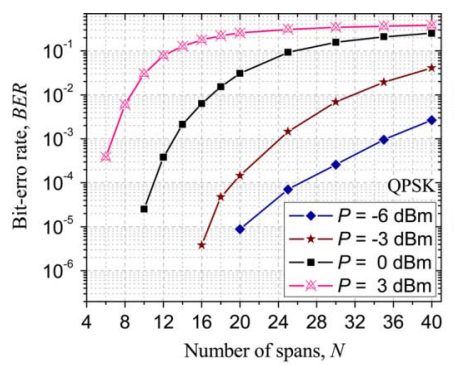

(a)

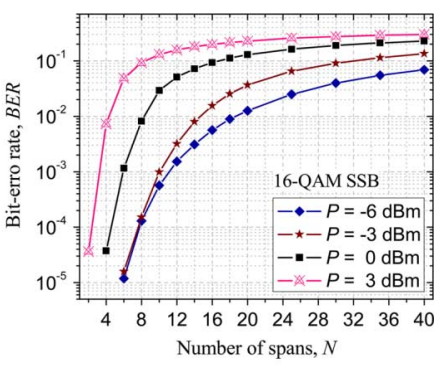

(b)
Fig. 5. Uncoded BER versus number of spans for SSB OFDM transmission and (a) QPSK, (b) 16-QAM modulation, for different launched powers $(P)$. (Color version available online at http://ieeexplore.ieee.org.)

not exhibit error-floor in the region of interest for $100-\mathrm{Gb} / \mathrm{s}$ Ethernet (below $10^{-12}$ ). The QPSK OFDM combined with LDPC coding provides the coding gain improvement of more than $2 \mathrm{~dB}$ over the LDPC coded RZ-OOK at BER of $10^{-8}$.

\section{PERformance ANALYSIS AND CONCLUSION}

The results of simulation in the presence of Kerr nonlinearities and ASE noise are given in Figs. 4 and 5. The dispersion map is composed of $N$ spans of length $L=120 \mathrm{~km}$, consisting of $2 L / 3 \mathrm{~km}$ of $D_{+}$fiber followed by $L / 3 \mathrm{~km}$ of $D_{-}$fiber, with precompensation of $-1600 \mathrm{ps} / \mathrm{nm}$ and corresponding postcompensation. $D_{+}$fiber has a dispersion of $20 \mathrm{ps} /(\mathrm{nm} \cdot \mathrm{km})$, a dispersion slope of $0.06 \mathrm{ps} /\left(\mathrm{nm}^{2} \cdot \mathrm{km}\right)$, an effective area equal to $110 \mu \mathrm{m}^{2}$, and loss equal to $0.19 \mathrm{~dB} / \mathrm{km}$. $D_{-}$fiber has a dispersion of $-40 \mathrm{ps} /(\mathrm{nm} \cdot \mathrm{km})$, a dispersion slope of $-0.12 \mathrm{ps} /\left(\mathrm{nm}^{2} \cdot \mathrm{km}\right)$, an effective area equal to $30 \mu \mathrm{m}^{2}$, and loss equal to $0.25 \mathrm{~dB} / \mathrm{km}$. The nonlinear Kerr coefficient is set to $2.6 \times 10^{-20} \mathrm{~m}^{2} / \mathrm{W}$ in both types of fibers. The dispersion map is chosen in such a way to increase the total transmission distance. For terrestrial applications, the total transmission distance is determined as $N \times 80 \mathrm{~km}$, since the dispersion compensating fiber is already included in the optical repeater.

The influence of Kerr nonlinearities is illustrated in Fig. 4 for QPSK SSB transmission [Fig. 4(a) and (b)], and 16-QAM SSB transmission [Fig. 4(c) and (d)]. The phase noise introduced by the self-phase modulation (SPM) causes the rotation of the constellation diagram [Fig. 4(a) and (c)]. By using the phase correction based on four pilot tones, the phase rotation due to SPM can be completely eliminated, as shown in Fig. 4(b) and (d). For worse dispersion maps, the larger number of pilot tones is required. Notice that after 20 spans $(2400 \mathrm{~km})$, the received constellation diagram for QPSK SSB transmission is still clear.

The BER curves for the uncoded 100-Gb/s OFDM SSB transmission using QPSK or 16-QAM are shown in Fig. 5(a) and (b), respectively. From Fig. 5(a) (for launched power of $-3 \mathrm{dBm}$ ) it can be concluded that $100-\mathrm{Gb} / \mathrm{s}$ transmission over $3840 \mathrm{~km}$ is possible using OFDM and LDPC codes [11] with threshold BER of $10^{-2}$, which cannot be achieved using the state-of-the art ETDM high-speed electronics operating at $100 \mathrm{~Gb} / \mathrm{s}$ [5].

In conclusion, we demonstrated that $100-\mathrm{Gb} / \mathrm{s}$ transmission may be realized by using the SSB OFDM with 64 subcarriers and the $25-\mathrm{GHz}$ bandwidth. OFDM is shown to be an efficient modulation format for long-haul transmission systems that provides a number of advantages: 1) increasing the transmission distance, 2) improvement of spectral efficiency, and 3) simplification of the dispersion compensation engineering. It is demonstrated by simulations that $100-\mathrm{Gb} / \mathrm{s}$ transmission over $3840 \mathrm{~km}$ can be achieved using single-sideband QPSK OFDM transmission, supplemented with LDPC codes, and commercially available components.

\section{REFERENCES}

[1] A. Zapata, "Next-generation 100-gigabit metro ethernet (100 GbME) using multiwavelength optical rings," J. Lightw. Technol., vol. 22, no. 11, pp. 2420-2434, Nov. 2004.

[2] M. Duelk, "Next Generation 100 G Ethernet," in Proc. ECOC 2005, Glasgow, Scotland, Sep. 25-29, 2005, Paper Tu3.1.2.

[3] G. Raybon, P. J. Winzer, and C. R. Doerr, " $10 \times 107-\mathrm{Gb} / \mathrm{s}$ electronically multiplexed and optically equalized NRZ transmission over 400 km," in Proc. OFC, 2006, Postdeadline Paper PDP32.

[4] M. Daikoku, "100 Gb/s DQPSK transmission experiment without OTDM for 100G Ethernet transport," in Proc. OFC, 2006, Postdeadline Paper PDP36.

[5] R. H. Derksen, "Integrated $100 \mathrm{~Gb} / \mathrm{s}$ ETDM receiver in a transmission experiment over 480 km DMF," in Proc. OFC, 2006, Postdeadline Paper PDP37.

[6] T. Lee, " $80^{+} \mathrm{Gb} / \mathrm{s}$ ETDM systems implementation: An overview of current technology," in Proc. OFC 2006, 2006, Paper OTuB3.

[7] R. Prasad, OFDM for Wireless Communications Systems. Boston: Artech House, 2004.

[8] Y. Wu and B. Caron, "Digital television terrestrial broadcasting," IEEE Commun. Mag., vol. 32, no. 5, pp. 46-52, May 1994.

[9] A. Kim, "60 GHz wireless communication systems with radio-overfiber links for indoor wireless LANs," IEEE Trans. Consum. Electron., vol. 50, no. 2, pp. 517-520, May 2004.

[10] I. B. Djordjevic, B. Vasic, and M. A. Neifeld, "LDPC coded orthogonal frequency division multiplexing over the atmospheric turbulence channel," in Proc. CLEO/QELS 2006, 2006, Paper CMDD5.

[11] I. B. Djordjevic, "Low-density parity-check codes for $40 \mathrm{~Gb} / \mathrm{s}$ optical transmission systems," IEEE J. Sel. Topics Quantum Electron., to be published. 